\title{
Effect of Mutation in Efflux Pump Regulatory Protein (MexR) of Pseudomonas aeruginosa: A Bioinformatic Study
}

\author{
Hamid Vaez (PhD) \\ Department of Microbiology, School \\ of Medicine, Zabol University of \\ Medical Sciences, Zabol, Iran \\ Vahid Vaez (DVM) \\ Department of Veterinary Medicine, \\ Islamic Azad University, Karaj branch, \\ Karaj, Iran \\ Farzad Khademi (PhD) \\ Department of Microbiology, School \\ of Medicine, Ardabil University of \\ Medical Sciences, Ardabil, Iran \\ Corresponding author: Farzad \\ Khademi \\ Email: f.khademi@arums.ac.ir \\ Tel: +989149679332 \\ Address: Department of \\ Microbiology, School of Medicine, \\ Ardabil University of Medical \\ Sciences, Ardabil, Iran \\ Received : 06 May 2017 \\ Revised: 29 Jun 2017 \\ Accepted: 29 Aug 2017
}

\begin{abstract}
Background and Objectives: Pseudomonas aeruginosa is an important nonfermenting gram-negative hospital-acquired pathogen. Treatment of $P$. aeruginosa infections has become more challenging due to overexpression of efflux pumps. The aim of the present study was to apply in silico analysis to evaluate the structure of the efflux pump regulatory protein, MexR, and impact of mutation on its stability and function.

Methods: Different bioinformatics tools including EXPASY, PROTEER, TECCOFFE, iStable, L-Mutant 2, STRING, ESPript, G0R IV, and PIB were used in the study.

Results: Aliphatic and instability indices were 104.15, and 46.52, respectively, indicating that the protein has a relatively short half-life. Most mutations decreased protein stability. Twenty-four mutations were identified as deleterious, with negative impact on the protein's function.

Conclusion: Determination of structure, variability, and function of MexR could be useful for modeling of treatment and control of multidrug resistant $P$. aeruginosa, with overexpressed efflux pump. We found that Mexk is a relatively unstable and conserved protein and the majority of mutations decrease its stability.

Keywords: Pseudomonas aeruginosa, MexR protein, Drug resistance, drug resistance multiple
\end{abstract}




\section{INTRODUCTION}

Pseudomonas aeruginosa is an important non-fermenting gram negative bacilli that can cause infections such as pneumonia, urinary tract infection, blood stream infection, meningitis, and skin infection, especially in immunocompromised patients (1). $P$. aeruginosa has a remarkable capacity to survive in adverse conditions $(2,3)$. According to previous studies, overexpression of efflux pumps genes has a crucial role in emergence of multi-drug resistance (MDR)-P. aeruginosa $(4,5)$. Several efflux pumps have been identified in $P$. aeruginosa. Based on their structure, source of energy and substrate, they have been classified into five families of resistance-nodulation-division (RND), ATPbinding cassette transporter family, major facilitator superfamily, multidrug and toxic compound extrusion, and small $\operatorname{MDR}(4,5)$. Active efflux pumps decrease intracellular concentration of different antibiotics by pumping out drugs, potentially leading to emergence of MDR- $P$. aeruginosa isolates. The RND family includes MexAB-oprM, MexCD-oprJ, MexEF-oprN, MexJK-oprM, and MexXY-oprM, which are targeted as substrate of different classes of antibiotics (4, 5). MexAB-oprM has three domains including membrane fusion protein, cytoplasmic membrane transporter, and outer membrane factor. MexAB-oprM operon is negatively regulated by the MexR, a 147 amino acid long regulatory protein encoded by the mex $\mathrm{R}$ gene, located at the upstream of the MexAB-oprM operon (6). Studies on 3D structure of the MexR showed that it is mainly consisted of $\alpha$ helices, and composes a triangular dimer with two DNA binding domains connected to each other $(4,5)$. In addition to their role in development of MDR- $P$. aeruginosa isolates, efflux pumps are also involved in survival and pathogenicity. For example, efflux pumps deletion notably reduces the ability of $P$. aeruginosa to invade epithelial cells (7). Beyond its role in epithelial cell invasion, efflux pumps have a critical role in cell-to-cell communication by quorum sensing signaling and biofilm formation. Biofilm formation by $P$. aeruginosa enables the bacteria to impair host's immunity. Therefore, disrupting biofilm formation in $P$. aeruginosa is of great importance (7). Amino acid substitutions can cause fundamental changes in normal proteins, affecting their stability, physicochemical properties and function. Hence, the use of bioinformatics tools has received considerable interest in recent years since they can predict the consequence of changes in normal protein, and contribute to drug design and development (8). As mentioned earlier, the MexAB-oprM efflux pump is one of the most important members of the RND family $(4,5)$ and its overexpression is mainly regulated by MexR. Therefore, the present study aimed to evaluate the effect of mutations on structure, stability, and function of MexR by using in silico analysis.

\section{MATERIAL AND METHODS}

We used $P$. aeruginosa PAO1 (NP_249115.1, NC_002516.2) as the reference strain, and its full MexR sequence was retrieved in FASTA format from the GenBank (https://www.ncbi.nlm.nih.gov/protein/). The MexR sequence was searched to find similar sequences using BlastP (https://blast.ncbi.nlm.nih.gov). Multiple alignments for the similar sequences were performed using T-COFFEE (http://www.tcoffee.org) and MEGA6 (9).

Primary structure of MexR was predicted using EXPASY (http://web.expasy.org/protparam) (10). Physiochemical properties such as molecular weight, atomic composition, chemical formula, amino acid composition, isoelectric point, aliphatic index, and approximate halflife were estimated (10). Secondary structure was predicted by ESPript 3.0 (http://espript.ibcp.fr) (11) and GOR IV (https://npsa-prabi.ibcp.fr). Three-dimensional structure was evaluated by SWISS MODEL (https://swissmodel.expasy.org/interactive) (10), and PDB file (1lnw) was saved for subsequent analysis. Three-dimensional structure of the reference protein (NP_249115.1) was obtained from the Protein Data Bank (http://www.rcsb.org). Transmembrane, intracellular and extracellular amino acids were predicted using PROTEER (http://wlab.ethz.ch/protter)(12). Interaction of MexR with other proteins was predicted using the STRING database (http://string-db.org). The STRING database provides a critical assessment and integration of protein-protein interactions, including direct (physical) and indirect (functional) associations. This database covers more than 2000 organisms, 
which has necessitated novel, scalable algorithms for transferring interaction information between organisms (13). Mutation of a single amino acid residue can cause some changes in a protein, which could result in the loss of protein function. We conducted a comprehensive search on popular databases including Web of Science, PubMed, Google scholar and Scopus to find reports on MexR mutation, using the following keywords: efflux pumps, MexR, MexAB-OprM and MDR $P$. aeruginosa. Finally, missense mutation reports were selected for the study. Three independent servers including I-Mutant2 (http://folding.biofold.org), iStable (http://predictor.nchu.edu.tw/iStable/), and PROVEAN (http://provean.jcvi.org) were used to evaluate effect of mutations on the protein. I-Mutant2 is an online program and support vector machines-based web server for prediction of protein stability changes upon single point mutation from the protein sequence and structure. According to the IMutant2 database, free energy change value (DDG) less than zero and more than zero can decrease and increase the stability of the protein, respectively. iStable provides an accurate approach for prediction of protein stability changes, using sequence information and prediction results from different element predictors (14).

\section{RESULTS}

Based on the results of BlastP (Table1), ten sequences with greatest identity were subjected to multiple alignments tools using the T-COFFEE program. MexR belongs to the Mar family of proteins that are involved in the emergence of MDR isolates. Figure 1 shows the results of multiple sequence alignments.
Primary structure analysis showed that the 147 amino acid long protein consists of Ala 10 (6.8\%), Arg 14 (9.5\%), Asn 5 (3.4\%), Asp 12 (8.2\%), Cys 3 (2.0\%), Gln 12 (8.2\%), Glu 10 $(6.8 \%)$, Gly $4(2.7 \%)$, His $6(4.1 \%)$, Ile 7 (4.8\%), Leu 23 (15.6\%), Lys 3 (2.0\%), Met 5 $(3.4 \%)$, Phe $4(2.7 \%)$, Pro $9(6.1 \%)$, Ser 4 (2.7\%), Thr $6(4.1 \%)$, Trp $0(0.0 \%)$, Tyr 1 $(0.7 \%)$, and Val $9(6.1 \%)$. The protein contains 2395 atoms, with the following chemical formula: $\mathrm{C}_{739} \mathrm{H}_{1207} \mathrm{~N}_{221} \mathrm{O}_{220} \mathrm{~S}_{8}$. The predicted isoelectric point and molecular weight were 5.64 and 16964.54 Da, respectively. The aliphatic index and instability index were estimated to be 104.15 and 46.52 , respectively, indicating that the protein has a relatively short half-life. The secondary structure of the protein was mainly alpha helix $(71.43 \%)$ and random coil (28.57\%) (Figure 2). The secondary structure of the protein was mainly composed of $\alpha$-helix sheets. The transmembrane, intracellular and extracellular amino acid prediction by the PROTEER server showed that MexR has no transmembrane residue. As shown in figure 3, the prediction revealed that the sequences with substitutions exhibit interactions similar to those of the reference strain. The following interactions were observed in the results: MexA (RND multidrug efflux membrane fusion protein precursor), amrB (multidrug efflux protein), rhlR (transcriptional regulator of RhlR necessary for transcriptional activation of the rhlAB genes), MexB (inner membrane transporter component of the MexAB-OprM efflux system that confers MDR), armR (antirepressor for MexR), MexF (RND multidrug efflux transporter), GyrA (DNA gyrase subunit $\mathrm{A}$ ), and $\mathrm{NfxB}$ (transcriptional regulator of resistance to quinolones).

Table1- Summary of BlastP for MexR ( $P$. aeruginosa)

\begin{tabular}{|c|c|c|c|c|c|c|}
\hline Accession & Identity & $\begin{array}{l}\text { Query } \\
\text { cover }\end{array}$ & $\begin{array}{l}\text { Total } \\
\text { score }\end{array}$ & $\begin{array}{l}\text { Max } \\
\text { score }\end{array}$ & Gaps & $\begin{array}{l}\text { Positive } \\
\text { number }\end{array}$ \\
\hline ANF04417.1 & $100 \%$ & $100 \%$ & 297 & 297 & $\begin{array}{l}0 / 147 \\
(0 \%)\end{array}$ & $\begin{array}{l}147 / 147 \\
(100 \%)\end{array}$ \\
\hline WP_043090638.1 & $99 \%$ & $100 \%$ & 296 & 297 & $\begin{array}{l}0 / 147 \\
(0 \%)\end{array}$ & $\begin{array}{l}146 / 147 \\
(99 \%)\end{array}$ \\
\hline WP_034039844.1 & $99 \%$ & $100 \%$ & 296 & 297 & $\begin{array}{l}0 / 147 \\
(0 \%)\end{array}$ & $\begin{array}{l}146 / 147 \\
(99 \%)\end{array}$ \\
\hline WP_034043761.1 & $99 \%$ & $100 \%$ & 296 & 297 & $\begin{array}{l}0 / 147 \\
(0 \%)\end{array}$ & $\begin{array}{l}146 / 147 \\
(99 \%)\end{array}$ \\
\hline WP_003114897.1 & $100 \%$ & $100 \%$ & 297 & 297 & $\begin{array}{l}0 / 147 \\
(0 \%)\end{array}$ & $\begin{array}{l}147 / 147 \\
(100 \%)\end{array}$ \\
\hline AAW82616.1 & $99 \%$ & $100 \%$ & 296 & 297 & $\begin{array}{l}0 / 147 \\
(0 \%)\end{array}$ & $\begin{array}{l}146 / 147 \\
(99 \%)\end{array}$ \\
\hline WP_046879890.1 & $99 \%$ & $100 \%$ & 295 & 297 & $\begin{array}{l}0 / 147 \\
(0 \%)\end{array}$ & $\begin{array}{l}146 / 147 \\
(99 \%)\end{array}$ \\
\hline WP_049249729.1 & $99 \%$ & $100 \%$ & 295 & 297 & $\begin{array}{l}0 / 147 \\
(0 \%)\end{array}$ & $\begin{array}{l}146 / 147 \\
(99 \%)\end{array}$ \\
\hline WP_034034038.1 & $99 \%$ & $100 \%$ & 296 & 297 & $\begin{array}{l}0 / 147 \\
(0 \%)\end{array}$ & $\begin{array}{l}146 / 147 \\
(99 \%)\end{array}$ \\
\hline WP_012613505.1 & $99 \%$ & $100 \%$ & 294 & 297 & $\begin{array}{l}0 / 147 \\
(0 \%) \\
\end{array}$ & $\begin{array}{l}146 / 147 \\
(99 \%)\end{array}$ \\
\hline
\end{tabular}


Figure1- Results of multiple alignments using T-COFFEE revealed that MexR is highly conserved. Yellow color shows variahle regions.



Figure2- Secondary structure prediction by ESPript depicted alpha helix regions in MexR

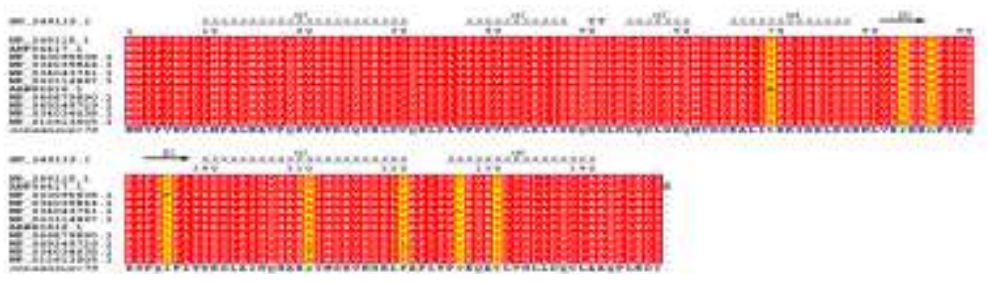

Figure 3- Schematic of protein-protein interactions predicted by STRING.

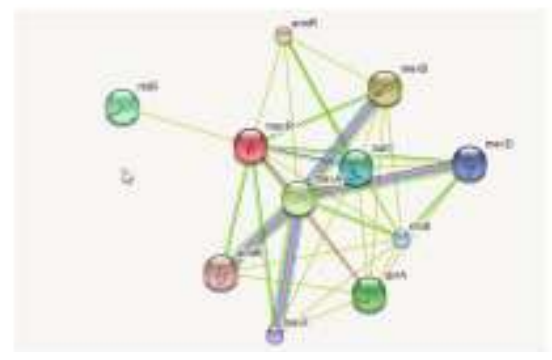

Colored lines between proteins indicate physical and functional interactions. Node size; small node, protein of unknown 3D structure, large node, some 3D structure is known or predicted. Colored nodes show first shell of interactors and white nodes show second shell of interactors. We found 46 amino acid substitutions at different positions in the online search for reports on mexR mutations (15-26). Results of analysis with IMutant2 showed that most of evaluated missense mutations decreased stability of the protein (Table 2). The mutations that stabilized the protein were seen at positions D8E, H107P, T130P, N53Y, N86I, and G58E.
According to the results obtained from iStable, alterations such as K44M, H107P, V132R, C30R, Q106R, N53Y, N53D, M10R, R78I, and $\mathrm{R} 21 \mathrm{~W}$ increased the stability of protein, while others decreased the stability (Table 2). Based on PROVEAN, substitutions at positions A66P, K44M, V126E, A66V, D8E, H107P, V132R, C30R, N79S, Q106R, L13M, and $\mathrm{R} 21 \mathrm{~W}$ were neutral and had no impact on protein function, while substitution at positions L45P, I46N, L57P, L57R, T69I, I72N, L75P, L75R, R83C, R91C, R91H，R114C，R70T, T130P, Q94P, R83H，G58E，L95F，T69I, N53Y, R78I, S88C, N86I, and R70W were deleterious (Table 2).

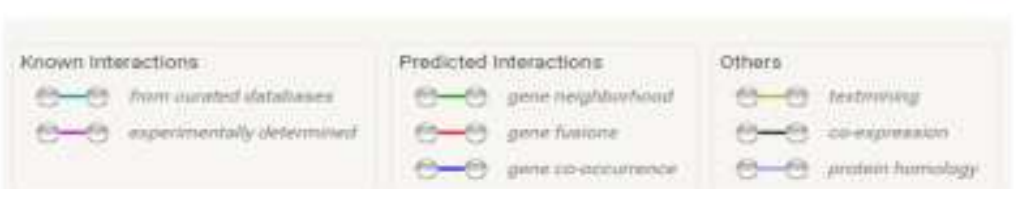


Table 2- Missense mutations and its impact on the protein

\begin{tabular}{|c|c|c|c|c|c|c|c|}
\hline \multirow[t]{2}{*}{ Position } & \multirow{2}{*}{$\begin{array}{l}\text { Amino acid } \\
\text { substitution }\end{array}$} & \multicolumn{2}{|l|}{ I Mutant 2} & \multicolumn{2}{|l|}{ i Stable } & \multicolumn{2}{|c|}{ PROVEAN } \\
\hline & & Stability & DDG & Stability & Score $^{\mathrm{a}}$ & Score $^{b}$ & Prediction \\
\hline 66 & A66P & Decrease & -0.84 & Decrease & 0.73 & -2.40 & Neutral \\
\hline 44 & K44M & Decrease & -0.74 & Increase & 0.84 & 0.16 & Neutral \\
\hline 126 & V126E & Decrease & -2.2 & Decrease & 0.67 & 2.38 & Neutral \\
\hline 66 & A66V & Decrease & 0.07 & Decrease & 0.80 & -2.36 & Neutral \\
\hline 8 & D8E & Increase & 0.05 & Decrease & 0.60 & 0.22 & Neutral \\
\hline 107 & H107P & Increase & 0.17 & Increase & 0.83 & 1.16 & Neutral \\
\hline 132 & V132R & Decrease & -2.1 & Increase & 0.83 & -0.58 & Neutral \\
\hline 45 & L45P & Decrease & -1.65 & Decrease & 0.66 & -3.04 & Deleterious \\
\hline 46 & I46N & Decrease & -1.69 & Decrease & 0.65 & -5.06 & Deleterious \\
\hline 57 & L57P & Decrease & -1.02 & Decrease & 0.80 & -6.6 & Deleterious \\
\hline 57 & L57R & Decrease & -1.20 & Decrease & 0.78 & -5.8 & Deleterious \\
\hline 69 & T69I & Decrease & -1.27 & Decrease & 0.80 & -3.9 & Deleterious \\
\hline 72 & I72N & Decrease & -0.60 & Decrease & 0.80 & -5.7 & Deleterious \\
\hline 75 & L75P & Decrease & -1.1 & Decrease & 0.81 & -6.5 & Deleterious \\
\hline 75 & L75R & Decrease & -2.16 & Decrease & 0.80 & -5.6 & Deleterious \\
\hline 83 & R83C & Decrease & -0.30 & Decrease & 0.72 & -7.4 & Deleterious \\
\hline 91 & R91C & Decrease & -0.38 & Decrease & 0.76 & -7.9 & Deleterious \\
\hline 91 & R91H & Decrease & -1.4 & Decrease & 0.80 & -5.0 & Deleterious \\
\hline 30 & C30R & Decrease & -0.02 & Increase & 0.71 & -1.86 & Neutral \\
\hline 114 & R114C & Decrease & -0.64 & Decrease & 0.71 & -4.68 & Deleterious \\
\hline 79 & N79S & Decrease & 0.08 & Decrease & 0.85 & -0.40 & Neutral \\
\hline 106 & Q106R & Decrease & -0.48 & Increase & 0.80 & -1.10 & Neutral \\
\hline 70 & R70T & Decrease & -0.33 & Decrease & 0.76 & -2.63 & Deleterious \\
\hline 130 & T130P & Increase & -0.59 & Decrease & 0.80 & -2.65 & Deleterious \\
\hline 94 & Q94P & Decrease & -1.14 & Decrease & 0.57 & -3.20 & Deleterious \\
\hline 13 & L13M & Decrease & -1.41 & Decrease & 0.84 & -0.68 & Neutral \\
\hline 21 & R21W & Decrease & -0.29 & Increase & 0.59 & -0.60 & Neutral \\
\hline 83 & R83H & Decrease & -1.24 & Decrease & 0.74 & -4.62 & Deleterious \\
\hline 58 & G58E & Increase & 0.60 & Decrease & 0.57 & -3.33 & Deleterious \\
\hline 95 & L95F & Decrease & -0.36 & Decrease & 0.90 & -3.05 & Deleterious \\
\hline 69 & T69I & Decrease & -1.27 & Decrease & 0.80 & -3.90 & Deleterious \\
\hline 70 & R70W & Decrease & -0.62 & Decrease & 0.62 & -4.83 & Deleterious \\
\hline 53 & N53Y & Increase & 0.05 & Increase & 0.62 & -2.93 & Deleterious \\
\hline 77 & G77A & Decrease & -1.77 & Decrease & 0.83 & 1.56 & Neutral \\
\hline 86 & N86I & Increase & 0.70 & Decrease & 0.82 & -4.82 & Deleterious \\
\hline 88 & S88C & Decrease & -0.86 & Decrease & 0.74 & -3.19 & Deleterious \\
\hline 63 & R63H & Decrease & -0.22 & Decrease & 0.85 & -1.81 & Neutral \\
\hline 53 & N53D & Decrease & -0.68 & Increase & 0.66 & -0.36 & Neutral \\
\hline 48 & E48K & Decrease & -0.05 & Decrease & 0.84 & 0.77 & Neutral \\
\hline 21 & R21G & Decrease & -1.73 & Decrease & 0.88 & -1.78 & Neutral \\
\hline 26 & S26G & Decrease & -1.71 & Decrease & 0.86 & -0.74 & Neutral \\
\hline 79 & N79G & Decrease & -1.61 & Decrease & 0.87 & 4.39 & Neutral \\
\hline 10 & M10R & Decrease & -0.30 & Increase & 0.50 & -0.69 & Neutral \\
\hline 78 & R78I & Decrease & -0.28 & Increase & 0.76 & -5.76 & Deleterious \\
\hline 103 & A103T & Decrease & -1.38 & Decrease & 0.83 & -1.34 & Neutral \\
\hline 106 & Q106H & Decrease & -1.09 & Decrease & 0.74 & -1.82 & Neutral \\
\hline
\end{tabular}

\section{DISCUSSION}

$P$. aeruginosa is considered as one of the most important hospital-acquired pathogens due to the resistance to multiple antibiotics and ability to survive on minimal nutritional requirements. Treatment of infections caused by this microorganism is becoming more challenging due to the resistance against multiple antibiotics (1-3). Efflux pumps are preponderant mechanisms of resistance because they extrude a wide range of substrates including penicillin, cephalosporin, carbapenems, monobactams, fluoroquinolones, and chemical disinfectants (27). The MexABOprM is an important efflux pump, negatively regulated by MexR (23).

In our study, the aliphatic and instability indices indicated that MexR is relatively unstable. The aliphatic index is directly related to the mole fraction of Ala, Ile, Leu, and Val (28). The aliphatic index of proteins from thermophilic bacteria was found to be significantly higher than that of ordinary proteins and hence, it can serve as a measure of thermos-stability of proteins (28). Mutations 
can affect protein folding, stability, and function, as well as protein-protein interactions. Moreover, it is essential to identify specific interaction partners for a protein to describe functions of the protein thoroughly $(10,29)$. In this study, the STRING program was used to predict protein-protein interactions, and the results showed that the interactions in the variants investigated were similar to that of the reference strain (data not shown). In our study, we used two independent programs to determine the effects of mutations on stability. Results of I mutant2 revealed that the majority of mutations decreased the stability except those at positions D8E, H107P, T130P, N53Y, N86I and G58E. Moreover, results of iStable showed that alterations at positions K44M, H107P, V132R, C30R, Q106R, N53Y, N53D, M10R, R78I and R21W increased the stability of the protein, while others decreased the stability. However, the results of the two programs were not entirely identical. This could be due to difference in the accuracy of the two programs $(77 \%$ vs. $80 \%)$ and in data set used by the tools. On the other hand, optimal stability of a protein relies on various thermodynamic factors. Several theories support the hypotheses that formation of stable molecules is a thermodynamically controlled process. However, the significance of negative entropy, chaperones, and oxidative potential should not be neglected (30).

We used PROVEAN to identify the effect of mutations on the protein's function. Of 46 amino acid substitutions, 24 mutations were identified as deleterious, and the remaining

\section{REFERENCES}

1. Vaez H, Faghri J, Nasr Esfahani B, Moghim S, Fazeli $\mathrm{H}$, Sedighi M, et al. Antibiotic Resistance Patterns and Genetic Diversity in Clinical Isolates of Pseudomonas aeruginosa Isolated From Patients of a Referral Hospital, Isfahan, Iran. Jundishapur $\mathrm{J}$ Microbiol. 2015;8(8):e20130. doi: 10.5812/jjm.20130v2.

2. Lister PD, Wolter DJ, Hanson ND. Antibacterialresistant Pseudomonas aeruginosa: clinical impact and complex regulation of chromosomally encoded resistance mechanisms. Clin Microbiol Rev. 2009; 22(4): 582-610. doi: 10.1128/CMR.00040-09.

3. Vaez H, Moghim S, Nasr Esfahani B, Ghasemian Safaei H. Clonal Relatedness among Imipenem-Resistant Pseudomonas aeruginosa Isolated from ICUHospitalized Patients. Crit Care Res Pract. 2015; 2015:983207. doi: 10.1155/2015/983207. ones were neutral. Most deleterious substations were related to residues 66-95. Regarding the secondary structure, 16 of 24 deleterious mutations were mapped at $\alpha-4$ and between $\alpha-4$ and $\alpha-5$. In the crystal structure of MexR, helices 1,4 and 5 are involved in dimerization, while $\alpha-4$ plays a crucial role in DNA binding (31). Studies of Andersen et al. and Saito et al. showed that mutations at $\mathrm{R} 83 \mathrm{H}, \quad \mathrm{R} 91 \mathrm{H}$, and $\mathrm{L} 95 \mathrm{~F}$ significantly overexpress efflux pump, suggesting that MexR impaired DNA binding activity (16, 22).

\section{CONCLUSION}

Although different bioinformatics tools are available for determination of sequence, structure and function of a protein, their outputs are not identical. Therefore, it is necessary to use multiple programs and combine the results for the final interpretation. We found that the results of iStable and PROVEAN are almost identical. In addition, the majority of mutations decrease the stability of the MexR protein, particularly those located at the $\alpha-4$ residue.

\section{ACKNOWLEDGEMENTS}

We would like to express our gratitude to owners of the online bioinformatics tools used in the study and the staff of Zabol University of Medical Sciences.

\section{CONFLICT OF INTEREST}

The authors declare no conflict of interest.

4. Dreier J, Ruggerone P. Interaction of antibacterial compounds with RND e ffl ux pumps in Pseudomonas aeruginosa. Front Microbiol. 2015; 6: 660. doi: 10.3389/fmicb.2015.00660.

5. Sun J, Deng Z, Yan A. Bacterial multidrug efflux pumps: mechanisms, physiology and pharmacological exploitations. Biochem Biophys Res Commun. 2014; 453(2): 254-67. doi: 10.1016/j.bbrc.2014.05.090.

6. Vaez H, Faghri J, Isfahani BN, Moghim S, Yadegari $\mathrm{S}$, Fazeli $\mathrm{H}$, et al. Efflux pump regulatory genes mutations in multidrug resistance Pseudomonas aeruginosa isolated from wound infections in Isfahan hospitals. Adv Biomed Res. 2014;3:117. doi: 10.4103/2277-9175.133183. 
7. Rasamiravaka T, Labtani Q, Duez P, El Jaziri M. The formation of biofilms by Pseudomonas aeruginosa: a review of the natural and synthetic compounds interfering with control mechanisms. Biomed Res Int. 2015; 2015: 759348. doi: 10.1155/2015/759348.

8. Teng S, Srivastava AK, Wang L. Sequence featurebased prediction of protein stability changes upon amino acid substitutions. BMC Genomics. 2010;11 (Suppl 2): S5. doi: 10.1186/1471-2164-11-S2-S5.

9. Tamura K, Stecher G, Peterson D, Filipski A, Kumar S. MEGA6: Molecular Evolutionary Genetics Analysis version 6.0. Mol Biol Evol. 2013; 30(12): 2725-9. doi: 10.1093/molbev/mst197.

10. Gasteiger E, Hoogland C, Gattiker A, Duvaud S, Wilkins MR, Appel RD. The Proteomics Protocols Handbook. Humana Press. 2005.

11. Robert X, Gouet P. Deciphering key features in protein structures with the new ENDscript server. Nucleic Acids Res. 2014;42(Web Server issue):W320-4. doi: 10.1093/nar/gku316.

12. Omasits U, Ahrens $\mathrm{CH}$, Muller S, Wollscheid B. Protter: interactive protein feature visualization and integration with experimental proteomic data. Bioinformatics. 2014; 30(6): 884-6. doi: 10.1093/bioinformatics/btt607.

13. Szklarczyk D, Franceschini A, Wyder S, Forslund K, Heller D, Huerta-Cepas J, et al. STRING v10: proteinprotein interaction networks, integrated over the tree of life. Nucleic Acids Res. 2015; 43(Database issue): D44752. doi: 10.1093/nar/gku1003.

14. Chen CW, Lin J, Chu YW. iStable: off-the-shelf predictor integration for predicting protein stability changes. BMC Bioinformatics. 2013; 14 Suppl 2: S5. doi: $10.1186 / 1471-2105-14-S 2-S 5$.

15. Adewoye L, Sutherland A, Srikumar R, Poole K. The mexR repressor of the mexAB-oprM multidrug efflux operon in Pseudomonas aeruginosa: characterization of mutations compromising activity. J Bacteriol. 2002; 184(15):4308-12. doi: $10.1128 / J B .184 .15 .4308$ 4312.2002 .

16. Andresen C, Jalal S, Aili D, Wang Y, Islam S, Jarl A, et al. Critical biophysical properties in the Pseudomonas aeruginosa efflux gene regulator MexR are targeted by mutations conferring multidrug resistance. Protein Sci. 2010; 19(4): 680-92. doi: 10.1002/pro.343.

17. Campo Esquisabel AB, Rodriguez MC, Campo-Sosa AO, Rodriguez C, Martinez-Martinez L. Mechanisms of resistance in clinical isolates of Pseudomonas aeruginosa less susceptible to cefepime than to ceftazidime. Clin Microbiol Infect. 2011; 17(12): 181722. doi: 10.1111/j.1469-0691.2011.03530.x.

18. Higgins PG, Fluit AC, Milatovic D, Verhoef J, Schmitz FJ. Mutations in GyrA, ParC, MexR and NfxB in clinical isolates of Pseudomonas aeruginosa. Int $\mathrm{J}$ Antimicrob Agents. 2003; 21(5): 409-13.

19. Hocquet D, Bertrand X, Kohler T, Talon D, Plesiat P. Genetic and phenotypic variations of a resistant Pseudomonas aeruginosa epidemic clone. Antimicrob Agents Chemother. 2003; 47(6): 1887-94. doi: 10.1128/AAC.47.6.1887-1894.2003.
20. Llanes C, Hocquet D, Vogne C, Benali-Baitich D, Neuwirth C, Plesiat P. Clinical strains of Pseudomonas aeruginosa overproducing MexAB-OprM and MexXY efflux pumps simultaneously. Antimicrob Agents Chemother. 2004; 48(5): 1797-802. doi: 10.1128/AAC.48.5.1797-1802.2004.

21. Quale J, Bratu S, Gupta J, Landman D. Interplay of efflux system, ampC, and oprD expression in carbapenem resistance of Pseudomonas aeruginosa clinical isolates. Antimicrob Agents Chemother. 2006; 50(5): 1633-41.

22. Saito K, Akama H, Yoshihara E, Nakae T. Mutations affecting DNA-binding activity of the MexR repressor of mexR-mexA-mexB-oprM operon expression. J Bacteriol. 2003;185(20): 6195-8. doi: 10.1128/JB.185.20.61956198.2003 .

23. Srikumar R, Paul CJ, Poole K. Influence of mutations in the mexR repressor gene on expression of the MexAMexB-oprM multidrug efflux system of Pseudomonas aeruginosa. J Bacteriol. 2000; 182(5): 1410-4.

24. Suman G, Khan M, Sabitha K, Jamil K. Mutation in mexR-gene leading to drug resistance in corneal keratitis in human. Indian J Exp Biol. 2006; 44(11): 929-36.

25. Tomas M, Doumith M, Warner M, Turton JF, Beceiro A, Bou G, et al. Efflux pumps, OprD porin, AmpC beta-lactamase, and multiresistance in Pseudomonas aeruginosa isolates from cystic fibrosis patients. Antimicrob Agents Chemother. 2010; 54(5): 2219-24. doi: 10.1128/AAC.00816-09.

26. Ziha-Zarifi I, Llanes C, Kohler T, Pechere JC, Plesiat P. In vivo emergence of multidrug-resistant mutants of Pseudomonas aeruginosa overexpressing the active efflux system MexA-MexB-OprM. Antimicrob Agents Chemother. 1999; 43(2): 287-91.

27. Alekshun MN, Levy SB. Molecular mechanisms of antibacterial multidrug resistance. Cell. 2007; 128(6): 1037-50. DOI:10.1016/j.cell.2007.03.004.

28. Idicula-Thomas S, Balaji PV. Understanding the relationship between the primary structure of proteins and their amyloidogenic propensity: clues from inclusion body formation. Protein Eng Des Sel. 2005; 18(4): 17580. DOI:10.1093/protein/gzi022.

29. Reva B, Antipin Y, Sander C. Predicting the functional impact of protein mutations: application to cancer genomics. Nucleic Acids Res. 2011; 39(17): e118. doi: $10.1093 /$ nar/gkr407.

30. Gummadi SN. What is the role of thermodynamics on protein stability? . Biotechnology and Bioprocess Engineering. 2003; 8(1): 9-18.

31. Lim D, Poole K, Strynadka NC. Crystal structure of the MexR repressor of the mexRAB-oprM multidrug efflux operon of Pseudomonas aeruginosa. J Biol Chem. 2002; 277(32): 29253-9. 